765 TREATMENT PATTERNS, REAL-WORLD OUTCOMES, AND RESOURCE USE IN PATIENTS WITH NON-MSI-HIGH OR MISMATCH REPAIR PROFICIENT ADVANCED ENDOMETRIAL CANCER

'S Kelkar, 'S Corman, ${ }^{2} \mathrm{C}$ Macahilig, ${ }^{3} \mathrm{~V}$ Prabhu*, ${ }^{4} \mathrm{~J} Z \mathrm{Zhang},{ }^{1} \mathrm{~N}$ Rusibamayila, ${ }^{2} \mathrm{~S}$ Odak, ${ }^{5} \mathrm{~L}$ Duska. ${ }^{1}$ OPEN Health, Evidence and Access, Bethesda, MD, USA; ${ }^{2}$ RTI-Health Solutions, Surveys and Observational Studies, Research Triangle, NC, USA; ${ }^{3}$ Merck and Co., Inc., Outcomes Research (Oncology-Cervical and Endometrial), Kenilworth, NJ, USA; ${ }^{4}$ Eisai, Inc. US HEOR and RWE, Wooddliff Lake, NJ, USA; ${ }^{5}$ University of Virginia Health System, Gynecologic Oncology Division Department of Obstetrics and Gynecology, Charlottesville, NC, USA

\subsection{6/ijgc-2021-ESG0.191}

Introduction/Background* Chemotherapy, the standard of care for patients with advanced endometrial cancer (aEC), has sub-optimal outcomes. In 2019, novel therapies specific to microsatellite instability (MSI)/mismatch repair (MMR) status changed the treatment landscape in the US. With sparse realworld outcomes data by MSI/MMR status, our study aimed to assess treatment patterns, real-world outcomes, and hospitalization stratified by treatment category, in aEC patients with non-MSI-high/MMR proficient (pMMR) tumors in the US.

Methodology Endometrial Cancer Health Outcomes (ECHO) is a multi-center, retrospective, chart review US study in which physicians consented to participate and provided de-identified data for adult women with inoperable non-MSI-high/pMMR aEC. Patients had $\geq 1$ prior systemic therapy and progressed between July 1, 2016 and June 30, 2019. Data collected included patient demographics, clinical characteristics, treatment category, clinical outcomes and hospitalization. KaplanMeier analyses were performed to estimate time to treatment discontinuation, real-world progression-free survival (rwPFS) and overall survival (OS), stratified by chemotherapy (CT) or hormonal therapy (HT). The study protocol was IRB approved.

Result(s)* The 139 patients included in this study were 64 years on average. About $64 \%$ were Caucasian, and 53\% had ECOG $\geq 2$. For $2^{\text {nd }}$-line therapy, 114 patients received CT, and 25 received HT, with a median follow-up of 9 and 8 months, respectively. Median time to discontinuation was 6 and 4 months in the HT and CT groups, respectively (table 1). Median OS since $2^{\text {nd }}$-line therapy initiation in the HT and CT groups was 9 and 10 months, respectively, median rwPFS was 6 and 5 months, respectively, and best overall response to $2^{\text {nd }}$-line therapy was $24 \%$ and $42 \%$, respectively. There were $16 \%$ patients with $\geq 1$ hospitalization (mean length of stay, 6

Abstract 765 Table 1 Time to treatment discontinuation, overall survival, and real-world progression free survival in non-MSI-High or pMMR aEC patients since initiation of 2 nd line therapy overall and stratified by treatment category

\begin{tabular}{|c|c|c|}
\hline & $\begin{array}{l}\text { Hormonal Therapy } \\
\qquad(N=25)\end{array}$ & $\begin{array}{l}\text { Chemotherapy } \\
(N=114)\end{array}$ \\
\hline Time to treatment discontinuation, median (95\% C1), (months) & $6.0(4.0,30.0)$ & $4.0\{3.0,5.0\}$ \\
\hline Probability at 6 months $(\%)$ & $66.7(50.2,88.5)$ & $53.5[45.1,63.5)$ \\
\hline Probability at 12 months $(\%)$ & $48.9(32.2,74.1)$ & $23.7(17,32.9)$ \\
\hline Probability at 24 months (\%) & $0(0,0)$ & $0(0,0)$ \\
\hline Overall survival, median (95\% Cl), (months) & $9.0(6.0, \mathrm{NE})$ & $10.0(8.0,14.0)$ \\
\hline Probability at 6 months $(\%)$ & $61.4(44.5,84.8)$ & $60.2(51.9,70)$ \\
\hline Probability at 12 months (\%) & $48.2(31.6,73.7)$ & $50.3(41.9,60.5)$ \\
\hline Probability at 24 months $(\%)$ & $43.9(27.6,69.7)$ & $41(32.7,51.3)$ \\
\hline Real-world progression-free survival, median (95\% C1), (months) & $5.5(3.0,29.0)$ & $5.0\{3.0,7.0\}$ \\
\hline Probability at 6 months $(\%)$ & $45.5(28.8,71.8)$ & 41.2 (33.1, 51.3) \\
\hline Probability at 12 months (\%) & $31.8(17.3,58.7)$ & $32.2(24.7,42.1)$ \\
\hline Probability at 24 months $(\%)$ & $31.8(17.3,58.7)$ & $22.3(15.7,31.5)$ \\
\hline
\end{tabular}

and 7 days for HT and CT groups, respectively), and 41\% of those had intensive care unit stay (mean, 2 and 5 days for HT and CT groups, respectively).

Conclusion* This study evaluated real-world treatment patterns, clinical outcomes, and hospitalization, stratified by treatment category in non-MSI-high/pMMR aEC patients in the US prior to July 2019. There continued to be significant clinical unmet need, indicating the need for novel therapies that delay progression, improve overall survival, and/or reduce hospitalization.

\section{TRANSVAGINAL ULTRASOUND FOR ASSESSING MYOMETRIAL INFILTRATION IN ENDOMETRIOD ENDOMETRIAL CANCER: LEARNING CURVE}

'BR Gastón Moreno*, ${ }^{2} J \mathrm{~L}$ Alcazar, 'JC Muruzábal Torquemada, 'Al Modroño Blanco, 'I Gómez Gutiérrez-Solana, ${ }^{1} \mathrm{C}$ Pérez Sanz, ${ }^{1} \mathrm{~A}$ García-Barberena Unzu, ${ }^{3} \mathrm{~N}$ Abián Franco. ${ }^{1}$ Complejo Hospitalario de Navarra, Obstetrics and Gynecology, Pamplona, Spain; ${ }^{2}$ Clínica Universidad de Navarra, Obstetrics and Gynecology, Pamplona, Spain; ${ }^{3}$ Hospital Reina Sofia, Obstetrics and Gynecology, Tudela, Spain

\subsection{6/ijgc-2021-ESGO.192}

Introduction/Background* Determining the degree of myometrial infiltration allows establishing the best therapeutic approach for each patient as it is an important factor in predicting nodal metastases.

Few prospective studies comparing the diagnostic performance of transvaginal ultrasound (TVS) and magnetic resonance imaging (MRI) in the preoperative local staging of endometrial carcinoma have been reported. In fact, a recent meta-analysis has shown that both techniques have similar diagnostic accuracy. However, to the best of our knowledge, there has been no prospective comparison of the diagnostic performance of TVS and MRI in the same group of patients with low-grade endometrial cancer.

The aim of this study was to analyse the learning curve when assessing myometrial infiltration by transvaginal ultrasound.

Methodology Observational prospective study performed at a single tertiary care centre from 2016 to 2020, comprising 156

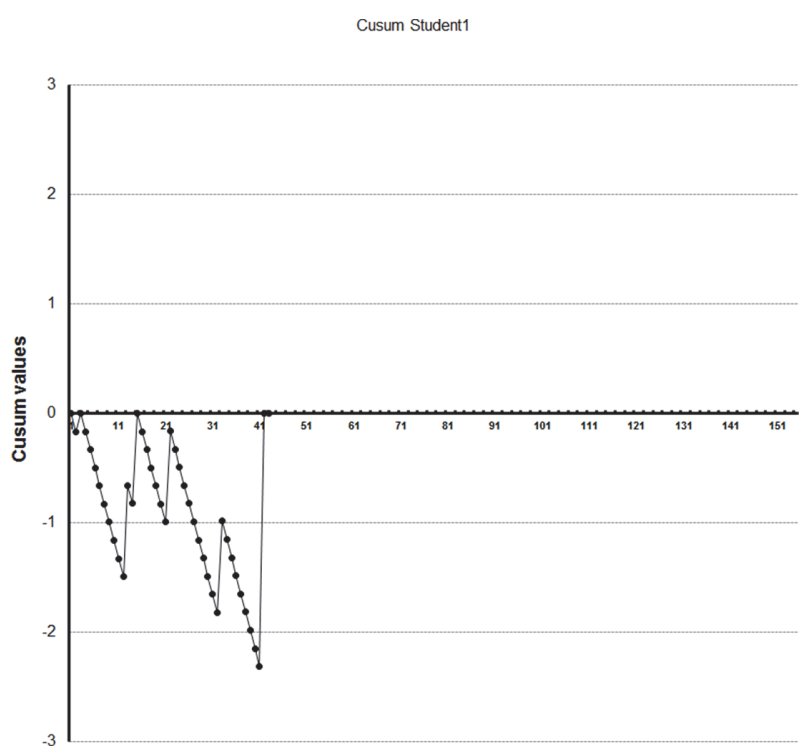

Abstract 767 Figure 1 